\title{
Surface expression of TTYH2 is attenuated by direct interaction with $\beta$-COP
}

\author{
Jiwon Ryu ${ }^{1, \#}$, Dong-Gyu Kim ${ }^{2, \#}$, Young-Sun Lee ${ }^{1}$, Yeonju Bae ${ }^{1}$, Ajung Kim ${ }^{2,3}$, Nammi Park ${ }^{1}$, Eun Mi Hwang ${ }^{2,3, *} \mathcal{E}^{2}$ \\ Jae-Yong Park ${ }^{1, *}$ \\ ${ }^{1}$ School of Biosystems and Biomedical Sciences, College of Health Sciences, Korea University, Seoul 02841, ${ }^{2}$ Korea Institute of Science and \\ Technology (KIST), Center for Functional Connectomics, Seoul 02792, ${ }^{3}$ KHU-KIST Department of Converging Science and Technology, \\ Graduate School, Kyung Hee University, Seoul 02447, Korea
}

TTYH2 is a calcium-activated, inwardly rectifying anion channel that has been shown to be related to renal cancer and colon cancer. Based on the topological prediction, TTYH2 protein has five transmembrane domains with the extracellular $\mathrm{N}$-terminus and the cytoplasmic C-terminus. In the present study, we identified a vesicle transport protein, $\beta-C O P$, as a novel specific binding partner of TTYH2 by yeast two-hybrid screening using a human brain cDNA library with the C-terminal region of TTYH2 (TTYH2-C) as a bait. Using in vitro and in vivo binding assays, we confirmed the protein-protein interactions between TTYH2 and $\beta$-COP. We also found that the surface expression and activity of TTYH2 were decreased by co-expression with $\beta$-COP in the heterologous expression system. In addition, $\beta$-COP associated with TTYH2 in a native condition at a human colon cancer cell line, LoVo cells. The over-expression of $\beta$-COP in the LoVo cells led to a dramatic decrease in the surface expression and activity of endogenous TTYH2. Collectively, these data suggested that $\beta$-COP plays a critical role in the trafficking of the TTYH2 channel to the plasma membrane. [BMB Reports 2019; 52(7): 445-450]

\section{INTRODUCTION}

TTYH genes are the human homolog of the Drosophila melanogaster gene tweety (1). Three members of the TTYH family (TTYH1-3) are reported to have large conductance chloride channels (2). Among these members of the TTYH

*Corresponding authors. Eun Mi Hwang, Tel: +82-2-958-7220; Fax: +82-2-958-7219; E-mail: emhwang@kist.re.kr; Jae-Yong Park, Tel: +82-2-3290-5637; Fax: +82-2-916-5943; E-mail: jaeyong68@korea.ac.kr ${ }^{\#}$ These authors contributed equally to this work.

https://doi.org/10.5483/BMBRep.2019.52.7.188

Received 14 August 2018, Revised 5 September 2018, Accepted 29 October 2018

Keywords: $\beta$-COP, Channel activity, LoVo cell, Surface expression, TTYH2 family, TTYH2 contains an acidic amino-acid cluster at the C-terminus, is predicted to interact with calcium ions, and displays calcium dependent chloride channel activity (2). TTYH2 is highly expressed in brain and testis, while it has low levels in the heart, ovary, and spleen (3). Based on the topological prediction, TTYH2 has five transmembrane domains with the extracellular $\mathrm{N}$-terminus and the cytoplasmic C-terminus (3).

The potential importance of the TTYH2 in causing diseases has been suggested by the fact that up-regulated expression of TTYH2 mRNA expression has been observed in renal cancer and colon cancer cells $(3,4)$. The analysis of comparative genomic hybridization also showed the chromosome region containing TTYH2, 17q24-q25, was highly amplified in various carcinomas (5). Furthermore, mRNAs of TTYH2 were highly expressed in human colon cancer cell lines LoVo, Caco-2 and DLD-1 (4). These findings imply that TTYH2 might have important roles in various cancers; however, physiological roles of TTYH2 are still unknown.

In general, activities of ion channels are regulated by protein-protein interactions (6). In this study, to better understand the function of TTYH2, we performed a yeast two-hybrid $(\mathrm{Y} 2 \mathrm{H})$ screening of a human brain cDNA library using the C-terminal cytosolic region of TTYH2 (TTYH2-C) as a bait. As a result, $\beta$-COP, a subunit of Coat Protein Complex I (COPI), was identified as a novel binding partner for TTYH2. Overexpression of $\beta$-COP decreased the surface expression and channel activity of TTYH 2 in a heterologous expression system and the LoVo cells. These data suggest that $\beta$-COP plays a critical role in the surface expression of the TTYH2 channel via direct protein-protein interaction.

\section{RESULTS}

TTYH2 associates with $\beta$-COP in vitro and in vivo To identify novel binding partners of TTYH2, Y2H screening was performed using a human fetal brain cDNA library with TTYH2-C (409-534 aa) as a bait (Fig. 1A). As a result, positive clones were isolated. Sequence analysis showed that one of the clones encoded a human $\beta$-COP, a subunit of the COPI complex (7). $\mathrm{Y} 2 \mathrm{H}$ assays were performed to confirm the 
A
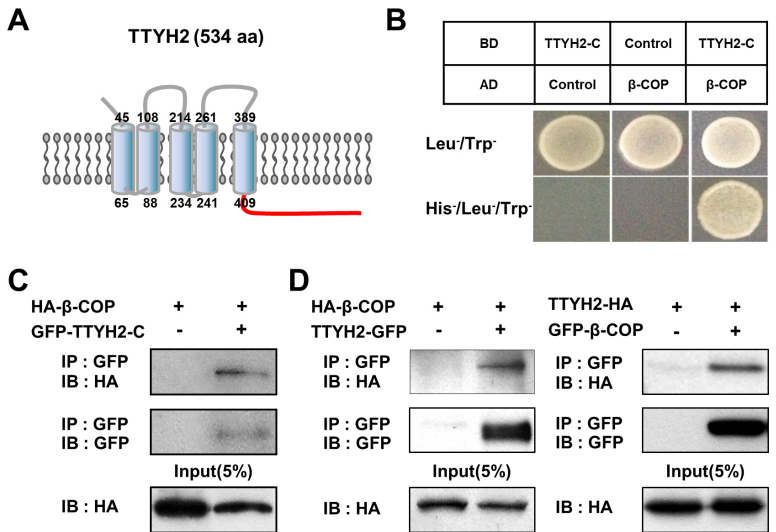

D

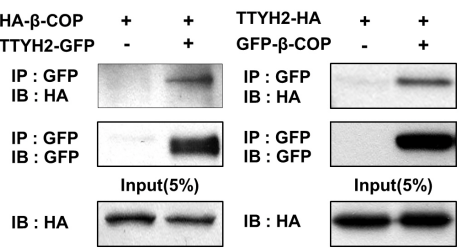

E

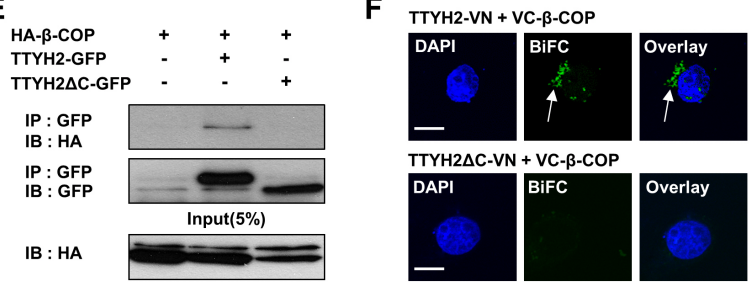

Fig. 1. $\beta$-COP directly interacts with the C-terminus of TTYH2 in vitro. (A) Schematic diagram of full-length TTYH2. C-terminus of TTYH2 is marked in red. The numbers represent the number of amino acids at the beginning and the end of each transmembrane domain of TTYH2. (B) Yeast two-hybrid (Y2H) assay showed that $\beta$-COP physically interacts with C-terminus of TTYH2 (TTYH2-C). (C) Co-immunoprecipitation (Co-IP) assay also showed HA- $\beta$-COP binds to GFP-TTYH2-C. (D) Reverse Co-IP of full-length $\beta$-COP and TTYH2 confirmed their interaction. (E) Co-IP assay showed $\beta$-COP interacts with TTYH2 but not with C-terminal lacking TTYH2 (TTYH2 $\triangle \mathrm{C}$ ). (F) BiFC assay was performed in VC- $\beta$-COP transfected COS-7 cells with TTYH2-VN or TTYH2 $2 \mathrm{C}-\mathrm{VN}$. The nuclei were indicated by DAPI staining. Fluorescence signals provide evidence of the proximity of the two proteins as indicated by the white arrow. Scale bars, $10 \mu \mathrm{m}$.

protein-protein interactions between TTYH2-C and $\beta$-COP (Fig. $1 \mathrm{~B})$, which clearly showed that $\beta$-COP directly binds to TTYH2-C. The positive yeast colony was also confirmed in $\mathrm{Y} 2 \mathrm{H}$ under non-permissive conditions in yeast media lacking Thr, Leu, and His $\left(\mathrm{TLH}^{-}\right)$, whereas the empty vector was not observed.

To confirm the interactions between $\beta$-COP and TTYH2-C, we constructed several expressing vectors containing $\mathrm{N}$-terminal green fluorescent protein (GFP)-tagged TTYH2-C (GFP-TTYH2-C), C-terminal GFP-tagged TTYH2 (TTYH2-GFP), $N$-terminal GFP-tagged $\beta$-COP (GFP- $\beta$-COP), $\quad N$-terminal HA-tagged $\beta$-COP (HA- $\beta$-COP), and C-terminal HA-tagged TTYH2 (TTYH2-HA). After the co-transfection of these expression vectors into COS-7 cells, co-immunoprecipitation (Co-IP) experiments showed that HA- $\beta$-COP was associated
A

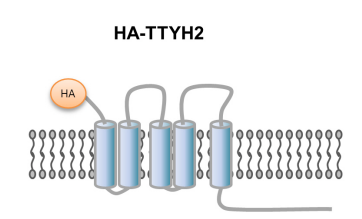

B

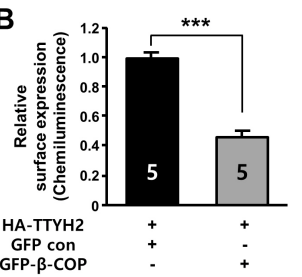

C
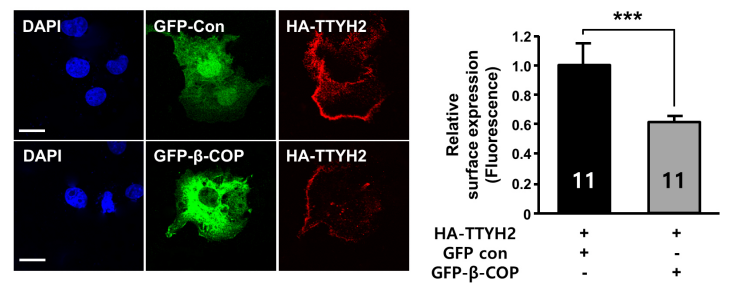

Fig. 2. $\beta$-COP suppresses the surface expression of $\mathrm{TTYH} 2$ in vitro. (A) Diagram of HA-tagged TTYH2 (HA-TTYH2) for surface expression assay. (B) Quantification of the cell surface expression of HA-TTYH2 in the presence of GFP or GFP- $\beta-$ COP was determined by chemiluminescence assay with an anti-HA antibody. (C) The surface expression of TTYH2 in the presence of GFP or GFP- $\beta$-COP was determined by immunocytochemistry assay with anti-HA antibody in non-permeabilized COS-7 cells. (D) The surface expression of HA-TTYH2 from (C) were quantified using Fluoview 1000 software. ${ }^{* * * P}<0.001$.

with GFP-TTYH2-C (Fig. 1C) and TTYH2-GFP (Fig. 1D, left panel). TTYH2-HA was also co-immunoprecipitated by GFP- $\beta$-COP (Fig. 1D, right panel). In addition, as shown in Fig. $1 \mathrm{E}, \mathrm{HA}-\beta$-COP was associated with TTYH2-GFP but not with TTYH2 $\triangle$ C-GFP (C-terminal GFP-tagged TTYH2 lacking C-terminal region), suggesting that an intracellular C-terminal region of TTYH2 is essential for the binding with $\beta$-COP.

Next, we examined the protein-protein interaction between TTYH2 and $\beta$-COP at the single-cell level using the bimolecular fluorescence complementation (BiFC) assay. The BiFC assay is a useful technique to visualize the proteinprotein interaction and its subcellular localization in a live cell $(8,9)$. We constructed three expressing vectors (TTYH2-VN, TTYH $2 \Delta \mathrm{C}-\mathrm{VN}$, and VC- $\beta-\mathrm{COP})$, which were the $\mathrm{N}$ - or C-terminal tagged with $\mathrm{N}$-terminal halve $(\mathrm{VN})$ or $\mathrm{C}$-terminal halve (VC) of the split Venus fluorescent protein. The BiFC analysis showed clear fluorescent signal in COS-7 cells co-expressing the TTYH2-VN and VC- $\beta$-COP (Fig. $1 \mathrm{~F}$, upper panel). In contrast, when TTYH $2 \Delta \mathrm{C}-\mathrm{VN}$ and $\mathrm{VC}-\beta-\mathrm{COP}$ were co-transfected into the cells, no fluorescence was detected (Fig. 1F, lower panel). As a negative control, when TTYH2-VN, TTYH $2 \Delta \mathrm{C}-\mathrm{VN}$, or VC- $\beta$-COP were individually transfected into the cells, fluorescence signals were not detected (data not shown). These data clearly showed the associations between TTYH2 and $\beta$-COP in live COS-7 cells. 
$\beta$-COP suppresses the surface expression and the activity of TTYH2 channels in heterologous expression system

Because $\beta$-COP protein has been shown to affect the surface expression of several ion channels $(10,11)$, we examined the effect of $\beta$-COP co-expression on the level of TTYH 2 on the plasma membrane. To measure the surface expression of TTYH2, the extracellular domain ( $\mathrm{N}$-terminus) of TTYH2 was tagged with an HA epitope (Fig. 2A). Both the luminescencebased cell surface assay (Fig. 2B) and the immunocytochemistrybased cell surface assay (Fig. 2C and D) were performed using anti-HA antibody. These data indicated that the surface expression level of TTYH2 was reduced by about $54 \%$ and $40 \%$ in COS- 7 cells by the presence of $\beta$-COP, respectively.

The effects of $\beta$-COP co-expression on TTYH 2 activity were also examined in whole-cell patch clamp recordings (Fig. 3). Whole-cell currents were measured during $1 \mathrm{sec}$ voltage-ramp pulses from -100 to $+100 \mathrm{mV}$, starting from a $0 \mathrm{mV}$ holding potential. As shown in Fig. $3 \mathrm{~A}$ and $\mathrm{B}$, background whole-cell currents were small in naïve COS-7 cells $(-11.73 \pm 1.58$ $\mathrm{pA} / \mathrm{pF}$ at $-100 \mathrm{mV}$ and $11.55 \pm 2.39 \mathrm{pA} / \mathrm{pF}$ at $+100 \mathrm{mV}, \mathrm{n}$ $=7$ ). However, currents from cells co-transfected with HA-TTYH2 and GFP control were dramatically increased over 2.5 fold $(-31.17 \pm 4.52 \mathrm{pA} / \mathrm{pF}$ at $-100 \mathrm{mV}$ and $36.28 \pm$ $4.76 \mathrm{pA} / \mathrm{pF}$ at $+100 \mathrm{mV}, \mathrm{n}=6$ ), with an almost linear current-voltage (I-V) relationship (Fig. $3 \mathrm{~A}$ and $3 \mathrm{~B})$. However, when GFP- $\beta$-COP was co-expressed with HA-TTYH2, the currents elicited by the same protocol in transfected cells were reduced to the same levels as the background currents $(-12.07 \pm 2.76 \mathrm{pA} / \mathrm{pF}$ at $-100 \mathrm{mV}$ and $14.62 \pm 2.8 \mathrm{pA} / \mathrm{pF}$ at $+100 \mathrm{mV}, \mathrm{n}=10$ ) (Fig. $3 \mathrm{~A}$ and $3 \mathrm{~B}$ ). These electrophysiological data strongly suggested that TTYH2-mediated chloride currents were suppressed by $\beta$-COP at the heterologous expression system.

\section{$\beta$-COP suppresses the surface expression and activity of} endogenous TTYH2 channels in LoVo cells

A previous study reported that mRNAs of TTYH2 were highly

A
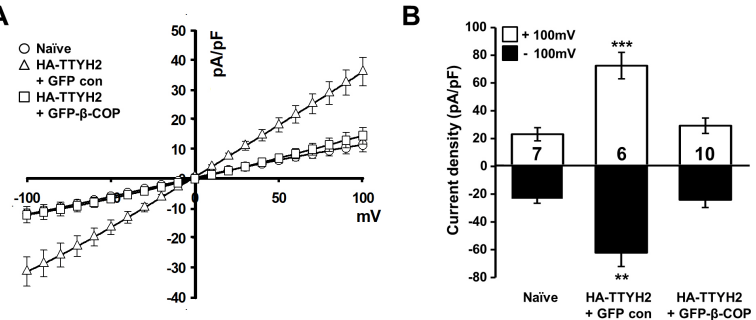

Fig. 3. $\beta-C O P$ decreases TTYH2-mediated whole-cell currents in vitro. (A) Whole-cell currents were measured in naïve COS-7 cells (circles, $\mathrm{n}=7$ ), in HA-TTYH2 and GFP co-expressed COS-7 cells (triangles, $\mathrm{n}=6$ ) or in HA-TTYH2 and GFP- $\beta$-COP co-expressed COS-7 cells (squares, $\mathrm{n}=10$ ). (B) Bar graph showed average current density at $-100 \mathrm{mV}$ and $+100 \mathrm{mV}$ as shown in (A). $* * * P<0.001$. expressed in human colon cancer cell lines (4). In agreement with this report, using RT-PCR analysis, we also found that TTYH 2 and $\beta$-COP are co-expressed in the human colon cancer line, LoVo cells (Fig. 4A). The interaction between TTYH2 and $\beta$-COP was confirmed in LoVo cells using Co-IP experiments with anti-TTYH2 antibody (Fig. 4B). Next, we examined whether the surface expression of endogenous TTYH 2 can be regulated by mCherry- $\beta$-COP overexpression in LoVo cell using immunocytochemistry (Fig. 4C and 4D). At

A
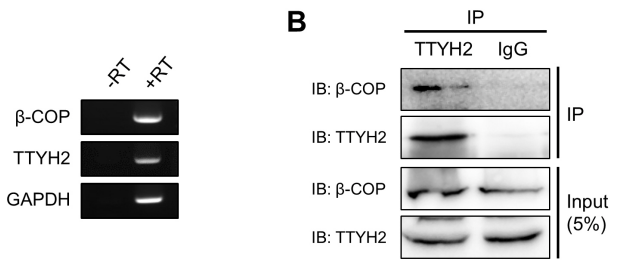

C

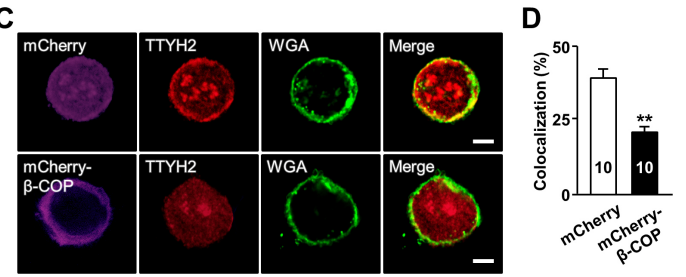

E

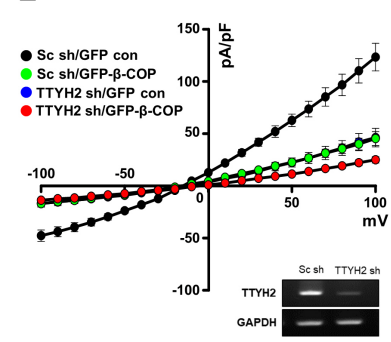

$\mathbf{F}$

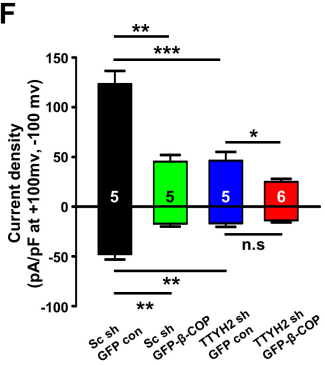

Fig. 4. Overexpressed $\beta$-COP decreases the surface expression and channel activity of TTYH2 in LoVo cells. (A) RT-PCR results showed that $\beta$-COP and TTYH2 were expressed in LoVo cells. $(-\mathrm{RT})$ without RT, (+RT) with RT. (B) Co-IP assay showed $\beta$-COP natively associated with TTYH2 in LoVo cells. (C) Overexpressed $\beta$-COP suppressed the surface expression of endogenous TTYH2 in LoVo cells. Wheat germ agglutinin (WGA) was used to delineate the plasma membrane of LoVo cells. The yellow colors in merged images indicate TTYH2 surface expression at the plasma membrane of the cells. Scale bar, 10 $\mu \mathrm{m}$. (D) Bar graph showed the percentage of colocalization with TTYH2 and WGA from individual cells. (E) Effect of $\beta$-COP overexpression on TTYH2-mediated whole-cell chloride currents in LoVo cells. Whole-cell currents were examined in Sc shRNA with GFP control (black circles, $\mathrm{n}=5$ ), Sc shRNA with GFP- $\beta-C O P$ (green circles, $\mathrm{n}=5$ ), TTHY2 shRNA with GFP control (blue circles, $\mathrm{n}=5$ ), and TTYH2 shRNA with GFP- $\beta$-COP (red circles, $\mathrm{n}=6$ ) transfected LoVo cells. The inset RT-PCR data showed the knocking down efficiency of TTYH2 shRNA in LoVo cells. (F) Bar graph showed average current density at $-100 \mathrm{mV}$ and +100 $\mathrm{mV}$ as shown in (E). ${ }^{*} \mathrm{P}<0.05,{ }^{*} * \mathrm{P}<0.01,{ }^{* * * P}<0.001$. 
the plasma membrane of control LoVo cell expressing mCherry, endogenous TTYH2 channels were mainly co-localized with the signals of wheat germ agglutinin (WGA)-647 dye, a maker of plasma membrane. However, mCherry- $\beta$-COP overexpression reduced endogenous TTYH2 level at the plasma membrane of LoVo cells (Fig. 4C and 4D). These results clearly showed that the surface expression of endogenous TTYH2 expression is reduced by the $\beta$-COP overexpression in LoVo cells.

Next, we examined the inhibitory effects of $\beta$-COP on endogenous $\mathrm{TTYH} 2$ activity in LoVo cells via whole-cell current recordings (Fig. 4E and 4F). To measure TTYH2mediated chloride currents in LoVo cells, we developed a TTYH2-specific short hairpin-forming interference RNA (shRNA) and validated the silencing efficiency of TTYH2 shRNA (inset in Fig. 4E). The TTYH2 shRNA expression significantly reduced both the outward and inward chloride currents of LoVo cells $(-16.77 \pm 3.6 \mathrm{pA} / \mathrm{pF}$ at $-100 \mathrm{mV}$ and $+46.22 \pm 8.86 \mathrm{pA} p \mathrm{pF}$ at $+100 \mathrm{mV}, \mathrm{n}=5$ ), compared to the currents of LoVo cells transfected with control scrambled shRNA (Sc shRNA) $(-47.75 \pm 5.42 \mathrm{pA} / \mathrm{pF}$ at $-100 \mathrm{mV}$ and $+123.28 \pm 13.19 \mathrm{pA} / \mathrm{pF}$ at $+100 \mathrm{mV}, \mathrm{n}=5$ ). This result clearly showed that TTYH2 contributed to the whole-cell chloride currents of LoVo cells. In addition, GFP- $\beta$-COP overexpression also dramatically reduced endogenous whole-cell chloride currents $(-17.13 \pm 2.92 \mathrm{pA} / \mathrm{pF}$ at $-100 \mathrm{mV}$ and $+45.27 \pm 6.69 \mathrm{pA} / \mathrm{pF}$ at $+100 \mathrm{mV}, \mathrm{n}=5$ ). The reducing effect of GFP- $\beta$-COP on whole-cell chloride currents was significantly abolished when GFP- $\beta$-COP were co-expressed with TTYH2 shRNA in LoVo cells $(-13.83 \pm 2.02 \mathrm{pA} / \mathrm{pF}$ at $-100 \mathrm{mV}$ and $+24.91 \pm 3.09 \mathrm{pA} / \mathrm{pF}$ at $+100 \mathrm{mV}, \mathrm{n}=5)$. Taken together, these results demonstrate that $\beta$-COP overexpression reduces the channel activity of endogenous TTYH2 channels, a major chloride channel in LoVo cells.

\section{DISCUSSION}

In this study, we found $\beta$-COP as a novel binding partner of TTYH2 (Fig. 1) and associated with endogenous TTYH2 in the human colon cancer line, LoVo cells (Fig. 4). The surface expression of the TTYH2 and TTYH2-mediated chloride currents in the heterologous and endogenous systems were suppressed by the overexpression of $\beta$-COP (Figs. 3 and 4). Taken together, these results clearly show that $\beta$-COP acts as a direct binding partner of the TTYH 2 and regulates the surface expression and channel activity of TTYH2.

The anterograde and retrograde transport of various ion channels are regulated by $\beta$-COP, a subunit of COP1 complex (7, 12-14). The surface expressions of CFTR and TREK-1 channels have also been shown to be increased by $\beta$-COP (10, 14). However, $\beta$-COP suppressed the surface expression of the TASK1 and ANO1 channels $(11,13)$. These studies showed that $\beta$-COP is involved in the surface expression of ion channels in the opposite direction. Therefore, more studies will be needed in order to gain understanding of how COP1 complex or $\beta$-COP specifically regulates the directional transport of many ion channels. Since it has been reported that proteins are recognized by the COP1 or $\beta$-COP subunit containing 1) KDEL motif $(15), 2) \mathrm{K}(X) \mathrm{KXX}$ motif (where $X$ represents any amino acid) (16), 3) di-basic motif (17), 4) RXR motif $(13,18)$, it will be necessary to determine the $\beta$-COP binding motif on C-terminus of TTYH 2 in the future.

In general, chloride channels are critical in maintaining cell volume in normal cells, and they also play important roles in migration and invasion of cancer cells (19). It has been reported that increased expressions and their dysfunctions of chloride channels have been implicated in different types of cancer $(20,21)$. Although the physiological role of TTYH2 has not yet clearly shown, the potential involvement of the TTYH2 in pathological conditions have been implicated in renal cell carcinoma and colon carcinoma $(3,4)$. In addition, small interfering RNA-mediated down-regulation of the TTYH2 reduces proliferation of colon cancer-derived Caco-2 and DLD-1 cells (4). These reports implied that activity of the TTYH2 channels may be important for the proliferation and tumorigenesis of cancer cells. Most of ion channels are localized at the plasma membrane for their physiological roles. Since the surface expression of ion channels is important for functional activity $(22,23)$, the interaction between $\beta$-COP and TTYH 2 may be critically involved in cancer proliferation and carcinogenesis, which calls for further studies.

Genome-wide proteomic studies to assess interaction networks of ion channels showed that ion channels can make numerous interactions with diverse signaling and scaffolding proteins (24-26). Therefore, it is plausible that ion channels also can play other roles at the intracellular organelles. Interestingly, recent study showed that TTYH1, another member of TTYH family, is involved in the maintenance of neural stem cell properties by increasing the Notch signaling pathway via protein-protein interaction with Rer1 (retention in endoplasmic reticulum sorting receptor 1 ) in the endoplasmic reticulum (27). This study clearly showed the role of TTYH1 chloride channel as a signaling protein at the intracellular organelle. Although the Rer1 do not bind with TTYH2 or TTYH3 (27), it is also possible that TTYH2 might be involved in intracellular signaling based on the retention of TTYH2 at the intracellular organelle via the protein-protein interaction with $\beta$-COP, which should be investigated in a future study.

In this study, for the first time, we found that $\beta$-COP was identified as having direct interaction with the C-terminal region of TTYH2. Furthermore, $\beta$-COP suppressed the surface expression and whole-cell currents of TTYH2 via the protein-protein interaction. These results suggest that the association with $\beta$-COP is critical for the surface expression of the TTYH2 channel and may help in increase understanding of the physiological roles of TTYH2 channels. 


\section{MATERIALS AND METHODS}

Detailed information is included in the Supplemental Material.

\section{Cell culture and transfection}

COS-7 and LoVo cells were maintained in an RPMI 1640 (Gibco) culture medium supplemented with $10 \%$ fetal bovine serum (Gibco) and $1 \%$ penicillin-streptomycin at $37^{\circ} \mathrm{C}$ in a $5 \% \mathrm{CO}_{2}$ environment. Transfection of expression vectors was performed using Lipofectamine 2000 (Invitrogen), according to the manufacturer's protocol.

\section{Construction of expression vectors and TTYH2 shRNA}

To construct expression vectors for human TTYH2 (NM 032646.5) and human $\beta$-COP (NM 016451), full-length genes of both were amplified by an RT-PCR and cloned into several destination vectors (pDEST-GFP-N, pDEST-GFP-C, pDEST-HA-N, pDEST-HA-C, pDEST-mCherry-N, pDEST-GBK, and pDEST-GAD) by Gateway Cloning System (Invitrogen). TTYH2 related deletion mutants were obtained by full-length cDNAs as templates using the EZchange ${ }^{\text {TM }}$ Site-directed Mutagenesis Kit (Enzynomics). The shRNA vector for human TTYH2 was constructed using the BLOCK-iT U6 RNAi Entry vector kit (Invitrogen). The target sequence of TTYH2 shRNA was 5'-GGATTATCTGGACGCTCTTGC-3' (CDS 1116-1136). The control Sc shRNA was constructed using the provided LacZ double-stranded control oligo.

\section{Yeast two-hybrid (Y2H) assay}

The DNA sequences encoding the TTYH2-C (409-534 aa; the C-terminal region of TTYH2) and the $\beta$-COP gene were cloned into the pDEST-GBK vector containing GAL4 DNA binding domain (BD) and the pDEST-GAD vector containing GAL4 activation domain (AD), respectively. To assess the proteinprotein interaction between TTYH2-C and $\beta$-COP, both recombinant plasmids were co-transformed into the yeast strain $\mathrm{AH} 109$, which is unable to synthesize histidine. In order for the yeast strains to grow on plates lacking histidine, protein interaction between $\mathrm{TTYH} 2-\mathrm{C}$ and $\beta$-COP was required. Protein interaction enables the yeast to make the His3 enzyme, thereby permitting histidine biosynthesis and growth on His minimal medium.

\section{ACKNOWLEDGEMENTS}

This work was supported by the National Research Foundation (NRF) of Korea (NRF2017M3A9C4092979).

\section{CONFLICTS OF INTEREST}

The authors have no conflicting interests.

\section{REFERENCES}

1. Campbell HD, Schimansky T, Claudianos C et al (1993) The Drosophila melanogaster flightless-I gene involved in gastrulation and muscle degeneration encodes gelsolin-like and leucine-rich repeat domains and is conserved in Caenorhabditis elegans and humans. Proc Natl Acad Sci U S A 90, 11386-11390

2. Suzuki $\mathrm{M}$ and Mizuno A (2004) A novel human $\mathrm{Cl}(-)$ channel family related to Drosophila flightless locus. J Biol Chem 279, 22461-22468

3. Rae FK, Hooper JD, Eyre HJ et al (2001) TTYH2, a human homologue of the Drosophila melanogaster gene tweety, is located on 17q24 and upregulated in renal cell carcinoma. Genomics 77, 200-207

4. Toiyama Y, Mizoguchi A, Kimura K et al (2007) TTYH2, a human homologue of the Drosophila melanogaster gene tweety, is up-regulated in colon carcinoma and involved in cell proliferation and cell aggregation. World J Gastroenterol 13, 2717-2721

5. Dohna M, Reincke M, Mincheva A et al (2000) Adrenocortical carcinoma is characterized by a high frequency of chromosomal gains and high-level amplifications. Genes Chromosomes Cancer 28, 145-152

6. Cho CH, Lee YS, Kim E et al (2015) Physiological functions of the TRPM4 channels via protein interactions. BMB Rep 48, 1-5

7. Bethune J, Wieland F and Moelleken J (2006) COPI-mediated transport. J Membr Biol 211, 65-79

8. $\mathrm{Hu} C D$, Chinenov $\mathrm{Y}$ and Kerppola TK (2002) Visualization of interactions among bZIP and Rel family proteins in living cells using bimolecular fluorescence complementation. Mol Cell 9, 789-798

9. Choi J, Jang Y, Kim H et al (2018) Functional roles of glutamic acid E143 and E705 residues in the N-terminus and transmembrane domain 7 of Anoctamin 1 in calcium and noxious heat sensing. BMB Rep 51, 236-241

10. Kim E, Hwang EM, Yarishkin O et al (2010) Enhancement of TREK1 channel surface expression by protein-protein interaction with beta-COP. Biochem Biophys Res Commun 395, 244-250

11. Lee $Y S$, Bae $Y$, Park N et al (2016) Surface expression of the Anoctamin-1 (ANO1) channel is suppressed by protein-protein interactions with beta-COP. Biochem Biophys Res Commun 475, 216-222

12. O'Kelly I, Butler MH, Zilberberg $N$ and Goldstein SA (2002) Forward transport. 14-3-3 binding overcomes retention in endoplasmic reticulum by dibasic signals. Cell 111, 577-588

13. Zuzarte M, Heusser K, Renigunta V et al (2009) Intracellular traffic of the $\mathrm{K}+$ channels TASK- 1 and TASK-3: role of $\mathrm{N}$ - and C-terminal sorting signals and interaction with 14-3-3 proteins. J Physiol 587, 929-952

14. Rennolds J, Tower C, Musgrove L et al (2008) Cystic fibrosis transmembrane conductance regulator trafficking is mediated by the COPI coat in epithelial cells. J Biol Chem 283, 833-839

15. Aoe T, Lee AJ, van Donselaar E et al (1998) Modulation of intracellular transport by transported proteins: insight from 
regulation of COPI-mediated transport. Proc Natl Acad Sci U S A 95, 1624-1629

16. Ma W and Goldberg J (2013) Rules for the recognition of dilysine retrieval motifs by coatomer. EMBO J 32, 926-937

17. Letourneur F, Gaynor EC, Hennecke S et al (1994) Coatomer is essential for retrieval of dilysine-tagged proteins to the endoplasmic reticulum. Cell 79, 1199-1207

18. Khalil H, Brunet A and Thibodeau J (2005) A three-aminoacid-long HLA-DRbeta cytoplasmic tail is sufficient to overcome ER retention of invariant-chain p35. J Cell Sci 118, 4679-4687

19. Peretti M, Angelini M, Savalli N et al (2015) Chloride channels in cancer: Focus on chloride intracellular channel 1 and 4 (CLIC1 AND CLIC4) proteins in tumor development and as novel therapeutic targets. Biochim Biophys Acta 1848, 2523-2531

20. Mao JW, Wang LW, Sun XR et al (2004) Volume-activated $\mathrm{Cl}$ - current in migrated nasopharyngeal carcinoma cells. Sheng Li Xue Bao 56, 525-530

21. Xu B, Mao J, Wang L et al (2010) ClC-3 chloride channels are essential for cell proliferation and cell cycle progression in nasopharyngeal carcinoma cells. Acta Biochim Biophys Sin (Shanghai) 42, 370-380

22. Steele DF, Eldstrom J and Fedida D (2007) Mechanisms of cardiac potassium channel trafficking. J Physiol 582, 17-26

23. Lee YS, Lee JK, Bae $Y$ et al (2016) Suppression of 14-3-3 $\gamma$-mediated surface expression of ANO1 inhibits cancer progression of glioblastoma cells. Sci Rep 6, 26413

24. Wang $X$, Wenable J, LaPointe $P$ et al (2006) Hsp90 cochaperone Aha1 downregulation rescues misfolding of CFTR in cystic fibrosis. Cell 127, 803-815

25. Perez-Cornejo P, Gokhale A, Duran C et al (2012) Anoctamin 1 (Tmem16A) Ca2 +-activated chloride channel stoichiometrically interacts with an ezrin-radixin-moesin network. Proc Natl Acad Sci U S A 109, 10376-10381

26. Chatr-Aryamontri A, Oughtred R, Boucher L et al (2017) The BioGRID interaction databse: 2017 update. Nucleic Acids Res 45, D369-D379

27. Kim J, Han D, Byun SH et al (2018) Ttyh1 regulates embryonic neural stem cell properties by enhancing the notch signaling pathway. EMBO Rep 19, e45472 